\title{
Combined Simulation of a Modified Piezoelectric Plate Transducer
}

\author{
M. Krause ${ }^{1}$, E. Starke ${ }^{1}$, G. Pfeifer ${ }^{1}$, T. Zahnert ${ }^{2}$ and W.-J. Fischer ${ }^{1}$ \\ ${ }^{1}$ Chair of Microsystems Technology, Technische Universität Dresden, Germany \\ Email: martin.krause@tu-dresden.de \\ ${ }^{2}$ Clinic of Otorhinolaryngology, Technische Universität Dresden, Germany
}

\begin{abstract}
:
It is common to use additional actuators to keep vibrating electromechanical systems without rigid connection to the inertial frame, e.g. cameras or hard disc drives, in position. In this paper we investigate an arrangement where a modified piezoelectric bending plate is used for position stabilization. In precision and microsystems engineering such plate transducers are used for transduction of volume flows or pressures. Other applications can be found in medical devices. The transducer is placed inside a closed capsule providing two air cavities in its front and back. A concentrated mass element connected to the plate supports the stabilization. Based on already existing network approaches the piezoelectric plate transducer model has been developed into a new multi domain network model. The network model further involves electrical, mechanical and acoustical properties of the enclosed transducer. Interactions between the transducer and the surrounding capsule have also been modeled in an acoustical network. The influence of size and numbers of holes in the plate on the electromechanical and acoustical properties of the transducer was investigated and optimized by network simulations.
\end{abstract}

Key words: modeling, unimorph, network methods, FE-methods, electromechanical transducer

\section{Introduction}

In microelectromechanical systems piezoelectric unimorph transducers are often used for electroacoustic sensor and actuator applications, e.g. microphones or speaker. This work presents a modified piezoelectric plate transducer as shown in Fig. 1.

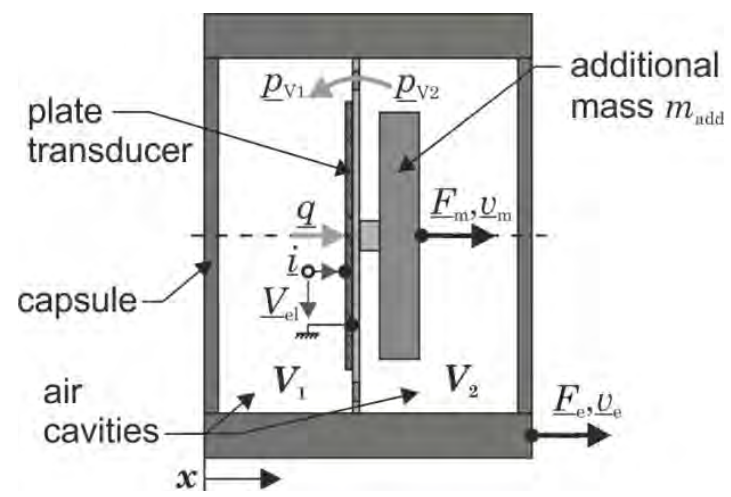

Fig. 1. Cross sectional view of the rotational symmetric unimorph inside a capsule including important physical parameters

The bendable carrier plate is fixed in a rotational symmetric capsule providing two cylindrical air cavities in the front and rear of the transducer. Such an arrangement is suited for vibration excitation or vibration damping of micromechanical objects attached to the capsule. Further the transducer employs a concentrated mass element. The mass increases the electromechanical performance of the transducer.

For analyzing the dynamic behavior of the transducer it has to be given respect to interactions between the electrical, mechanical and acoustical system. An applied electrical voltage at the piezoelectric element causes a mechanical displacement as well as an acoustic difference pressure $\underline{p}_{\mathrm{V} 2}-\underline{p}_{\mathrm{V} 1}$ across the unimorph. The acoustic properties of the transducer can be adjusted by perforating the carrier plate between the piezoelectric disc and its edge. The perforation allows an air exchange through the plate and decreases the pressure in the capsule. The mechanical and electrical properties are influenced by the perforation as well. A good acoustic behavior of the transducer, e.g. a minimal difference pressure, comes to the expense of the electromechanical actuating properties. The following sections consider the influence of the perforation on the electromechanical and acoustical properties of the plate transducer. 
Therefore the arrangement in Fig. 1 is analyzed using network and FE-methods.

\section{Network approach of the encapsulated plate transducer}

Currently there does not exist any network model of a circular plate transducer with respect to the electrical, mechanical and acoustical domain. Hence a multi domain network model was developed based on existing network approaches.

The plate transducer as well as the surrounding capsule was transferred into a network model using the methods of the "Combined Simulation" described by Starke et al. [1, 2]. This method connects the advantages of network and FE-methods. Network methods enable a fast simulation of an electromechanical system ensuring an efficient design process. Further electrical engineers are familiar with circuit simulations. The values of the network elements are determined using static FE-simulation experiments. This procedure allows a faster simulation of the system than a harmonic or transient FEanalysis. The network model is valid up to the first resonance frequency which is sufficient.

The development of a simulation model of the encapsulated plate transducer requires to define main system points and affecting physical parameters to be modeled. These physical parameters shown in Fig. 1 can be divided into electrical, mechanical and acoustical ones. Electrical parameters are the voltage $\underline{V}_{\mathrm{el}}$ and current $\underline{i}$ at the piezoelectric element. The plate transducer operates as an electromechanical actuator. Therefore the electrical current is faced in the direction as it would be for an electrical source. The capacity $C_{\mathrm{b}}$ of the piezoelectric element is modeled, too. Mechanical system points are the velocities $\underline{v}_{\mathrm{m}}$ and $\underline{v}_{\mathrm{e}}$ as well as the forces $\underline{F}_{\mathrm{m}}$ and $\underline{F}_{\mathrm{e}}$ in the middle of the plate transducer and the capsule (see Fig. 1). These mechanical across and flow quantities are pointed in the same positive direction ( $x$ direction). In contrary the acoustic difference pressure $\underline{p}_{\mathrm{pl}}=\underline{p}_{\mathrm{V} 2}-\underline{p}_{\mathrm{V} 1}$ across the plate transducer and the acoustic volume flow $\underline{q}$ are pointed in opposite directions. The volume flow is as positive directed as the mechanical quantities. The difference pressure is faced in the negative direction. Thus a positive volume flow causes a positive difference pressure $\underline{p}_{\mathrm{pl}}$.

\section{Basic transducer network models}

Each of the following approaches simulates a fraction of the dynamic behavior of the transducer.

Fig. 2 shows the electromechanical network model of a piezoelectric disc provided by Lenk et al. [3]. It incorporates the mechanical compliance $n_{\mathrm{k}}$ and the effective vibrating mass $m$ of the disc as well as its clamped electrical capacity $C_{\mathrm{b}}$. The connection between both domains is realized by the electromechanical transduction factor $Y$ according to the equations in the box. In network analyzer programs $Y$ can be described using dependent voltage and current sources.

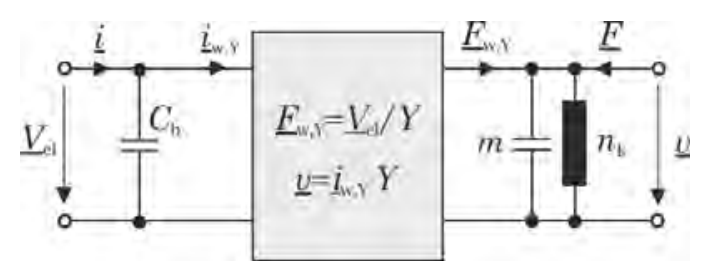

Fig. 2 Electrical-mechanical network model of a piezoelectric disc

Lenk et al. [3] provide a circuit for modeling the acoustic-mechanical properties of circular bendable plates (Fig. 3), too. The network model was extended by the mass fractions $m_{\mathrm{m}}$ and $m_{\mathrm{e}}$ to take account for mechanical resonance effects. The element $n_{\mathrm{k}}$ models the mechanical compliance of the unimorph. Mechanical and acoustical quantities are connected by the effective area $A_{\text {eff }}$ of the bending plate. This area corresponds to that area in case the bending plate would be an ideal piston.

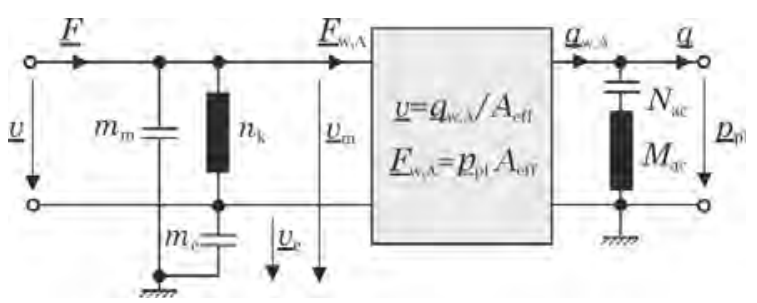

Fig. 3 Mechanical-acoustical network model of a circular bending plate

The network model in Fig. 3 consists of compliant and mass elements in its acoustic domain, too. These elements $N_{\mathrm{ac}}$ and $M_{\mathrm{ac}}$ take account for the ring mode vibration of the transducers shown in Fig. 4. Compared to the normal model vibration the ring mode vibration provides a volume flow $\underline{q}$ for an applied difference pressure $\underline{p}_{\mathrm{pl}}$ across the plate even in case of a fixed center and edge. 
normal mode vibration

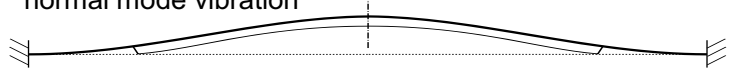

ring mode vibration

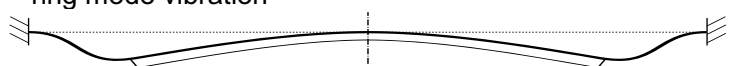

Fig. 4 Schematic of the two vibration modes of the unimorph bending plate

The network model shown in Fig. 5 has been provided by Starke et al. [2]. It simulates the electroacoustic behavior of a circular unimorph plate transducer. The model connects the electrical and acoustic domain and allows simulating the difference pressure $\underline{p}_{\mathrm{pl}}$ across the plate transducer for an applied voltage load $\underline{V}_{\mathrm{el}}$ at the piezoelectric element. The network does not include the affecting mechanical parameters of unimorph. Hence mechanical quantities cannot be simulated by this network.

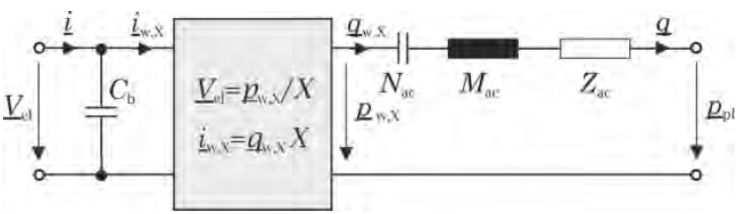

Fig. 5 Electrical-acoustical network model of a unimorph bending plate

Multi domain network model of the unimorph bending plate

The superposition of the approaches in Fig. 2-5 provides the three domain network of the unimorph bending plate shown in Fig. 6 .

The impedances $h_{\mathrm{bp}}$ and $Z_{\mathrm{ac}}$ have been added to the model to include material damping effects. The impedance values are calculated using the quality factors $Q$ of parallel and series resonance circuits. The equation has to be applied

$$
Q_{\mathrm{bp}}=h_{\mathrm{bp}} \cdot \sqrt{\frac{n_{\mathrm{k}}}{m_{\mathrm{m}}}} \text { and } Q_{\mathrm{ac}}=\frac{1}{Z_{\mathrm{ac}}} \cdot \sqrt{\frac{M_{\mathrm{ac}}}{N_{\mathrm{ac}}}} .
$$

Typical values of the quality factor for clamped plates are $Q=50 \ldots 80$ [2]. The additional mass connected to the middle of the transducer is modeled as mass element $m_{\text {add }}$. As already mentioned, the network approach in Fig. 6 is valid up to the first mechanical and acoustic resonance frequencies. Furthermore, the model does not take account for the velocity of sound inside the encapsulation. Hence the network model is valid for small dimensions of the unimorph relatively to the acoustic wavelength $\lambda$. Under the assumption of a plate diameter $r_{\mathrm{pl}}$, representing the largest dimension it has to be applied

$$
2 \cdot r_{\mathrm{pl}} \ll \lambda=\frac{c}{f} .
$$

In addition mechanical displacements and acoustic volume flows, respectively, have to be small compared to the dimensions of the plate transducer.

\section{Modeling of the acoustics}

The acoustic network shown in Fig. 7 has been proven suitable for modeling the perforation in the carrier plate of the transducer. It consists of acoustic compliances $N_{\mathrm{ac}, \mathrm{V} 1 / 2}$ for simulating air compression in the cavities $V_{1}$ and $V_{2}$ in front and rear of the plate. Acoustic mass elements $M_{\mathrm{ac}, \mathrm{V} 1 / 2}$ simulate the vibrating air volumes in front and rear of the plate, accordingly.

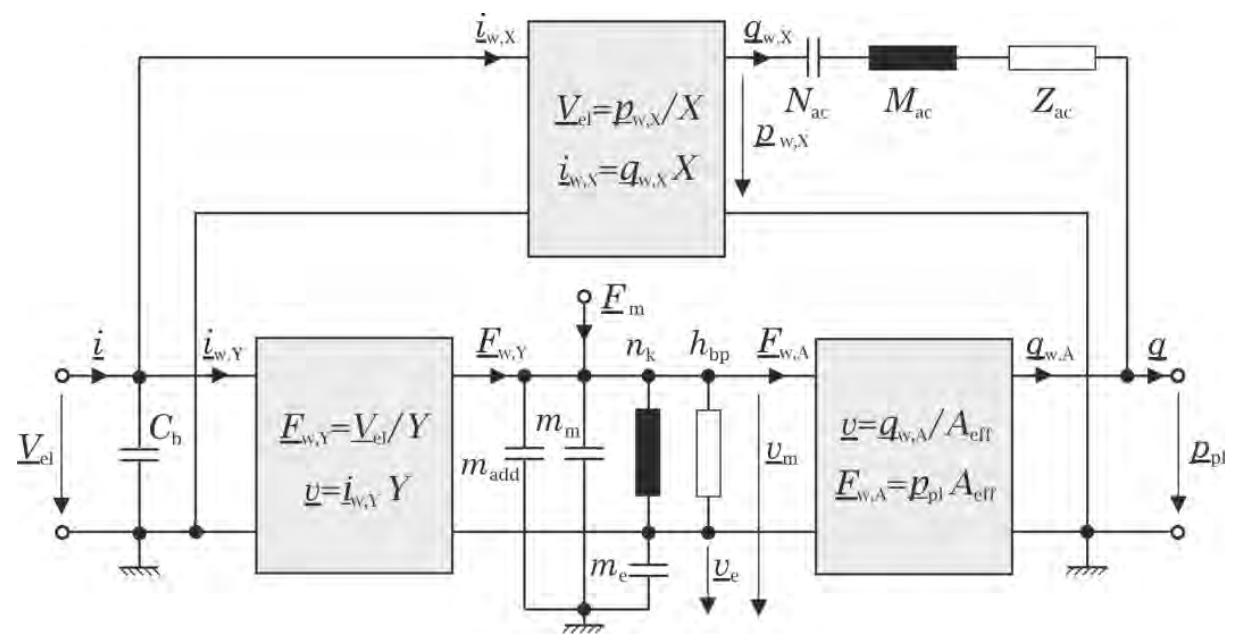

Fig. 6 Three-port multi domain network model of the unimorph bending plate 


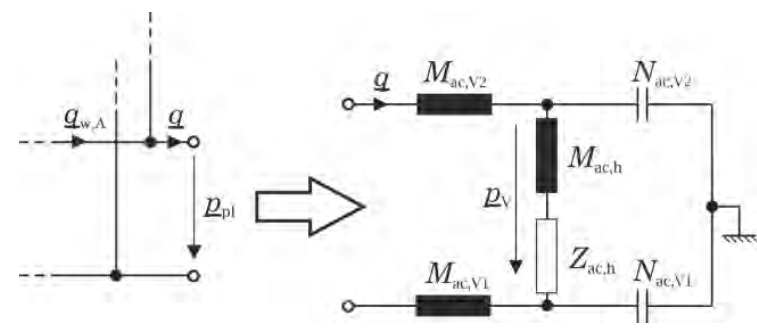

Fig. 7 Network of the acoustic behavior of the perforation and the air cavities

The air flow through the holes in the carrier plate is modeled as an acoustic mass element $M_{\text {ac,h }}$, too. In addition the acoustic impedance $Z_{\text {ac,h }}$ takes account for damping effects of the perforation. Fig. 7 shows a schematic allocation approach of the acoustic network elements to the acoustic behavior of the plate transducer and the capsule calculated by FE-methods.

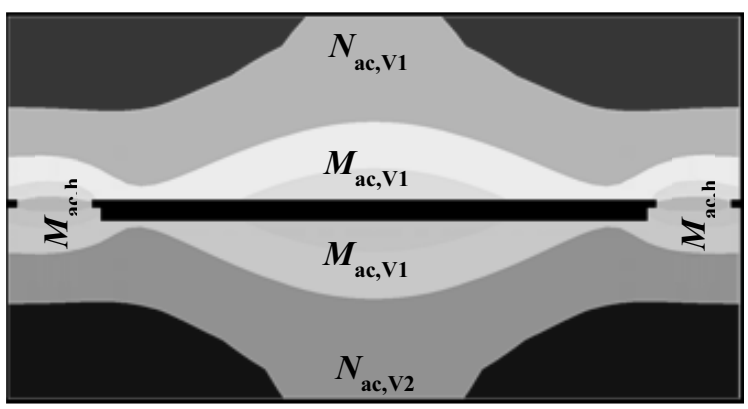

Fig. 8 FE-simulated pressure distribution inside the air cavities and allocation of the acoustic network elements

It is not possible to allocate the network elements clearly to the pressure distribution. For this reason the acoustic network assumes a constant acoustic pressure in the air cavities as well as no air compression inside the holes of the bending plate.
Determination of network parameters using FE-simulation experiments

According to the methods of the "Combined Simulation" the elements of the network model were determined using FE-methods. For this purpose an FE-model of the transducer has been implemented in ANSYS $尺$ (Release 13.0). Afterwards, network elements of the three domain network model in Fig. 6 were determined using static FE-experiments $(f=0 \mathrm{~Hz})$ and modal analyses respectively. All of the network elements can be calculated separately by choosing the right load and boundary conditions.

An example is following given by the determination of $n_{\mathrm{K}}$. In a static analysis the admittances of the parallel mass elements are large $\left(1 / j \omega m_{\mathrm{m} / \mathrm{e}} \rightarrow \infty\right)$, the impedance of the series mass $j \omega M_{\mathrm{ac}} \rightarrow 0$ is small, respectively. Accordingly all mass elements are negligible. Further the acoustical and electrical domain are short-circuit $\left(p_{\mathrm{pl}}=0 \mathrm{~Pa}, V_{\mathrm{el}}=0 \mathrm{~V}\right)$. Because of

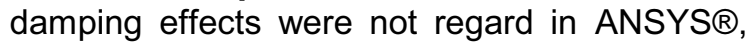
the element $n_{\mathrm{K}}$ has been isolated from the other circuit elements. For a force load of $F_{\mathrm{m}}=1 \mathrm{~N}$ into the middle of the plate transducer the compliance can be calculated using the displacement reaction $\xi_{\mathrm{m}}$ at this point by

$$
n_{\mathrm{K}}=\frac{\xi_{\mathrm{m}}}{F_{\mathrm{m}}} \text {. }
$$

Table 1 shows a summary of all FE-simulation experiments. The table opposes necessary load and boundary conditions to the equations for calculating the network elements of the plate transducer.

Table 1 Load and boundary conditions of the FE-simulation experiments for determining the network elements

\begin{tabular}{|c|c|c|c|}
\hline equation & input parameter & output parameter & boundary conditions \\
\hline \multicolumn{4}{|c|}{ static experiments $\left(v_{\mathrm{e}}=0 \mathrm{~m} / \mathrm{s}, f=0 \mathrm{~Hz}\right)$} \\
\hline$N_{\mathrm{ac}}=V / p_{p l}$ & $p_{\mathrm{pl}}$ & V & $v_{\mathrm{m}}=0 \mathrm{~m} / \mathrm{s}, V_{\mathrm{el}}=0 \mathrm{~V}$ \\
\hline$A_{\text {eff }}=F / p_{\mathrm{pl}}$ & $p_{\mathrm{pl}}$ & $F$ & $v_{\mathrm{m}}=0 \mathrm{~m} / \mathrm{s}, V_{\mathrm{el}}=0 \mathrm{~V}$ \\
\hline$X=-N_{\mathrm{ac}} \cdot V_{\mathrm{el}} / V$ & $V_{\mathrm{el}}$ & $V$ & $v_{\mathrm{m}}=0 \mathrm{~m} / \mathrm{s}, p_{\mathrm{pl}}=0 \mathrm{~Pa}$ \\
\hline$Y=-V_{\mathrm{el}} / F$ & $V_{\mathrm{el}}$ & $F$ & $v_{\mathrm{m}}=0 \mathrm{~m} / \mathrm{s}, p_{\mathrm{pl}}=0 \mathrm{~Pa}$ \\
\hline$C_{\mathrm{b}}=Q / V_{\mathrm{el}}$ & $V_{\mathrm{el}}$ & $Q$ & $v_{\mathrm{m}}=0 \mathrm{~m} / \mathrm{s}, p_{\mathrm{pl}}=0 \mathrm{~Pa}$ \\
\hline$n_{\mathrm{k}}=\xi_{\mathrm{m}} / F$ & $\xi_{\mathrm{m}}$ & $F$ & $p_{\mathrm{pl}}=0 \mathrm{~Pa}, V_{\mathrm{el}}=0 \mathrm{~V}$ \\
\hline \multicolumn{4}{|c|}{ modal analyses } \\
\hline$m_{m}=\left[\left(2 \pi f_{0, \mathrm{~mm}}\right)^{2} \cdot n_{k}\right]^{-1}$ & & $f_{0, \mathrm{~mm}}$ & $\underline{v}_{\mathrm{e}}=0 \mathrm{~m} / \mathrm{s}$ \\
\hline$m_{e}=\left[\left(2 \pi f_{0, \mathrm{me}}\right)^{2} \cdot n_{k}\right]^{-1}$ & & $f_{0, \mathrm{me}}$ & $\underline{v}_{\mathrm{e}}=\underline{v}_{\mathrm{m}}=0 \mathrm{~m} / \mathrm{s}$ \\
\hline$M_{\mathrm{ac}}=\left[\left(2 \pi f_{0, \mathrm{ac}}\right)^{2} \cdot N_{\mathrm{ac}}\right]^{-1}$ & & $f_{0, \mathrm{ac}}$ & $\underline{v}_{\mathrm{m}}=0 \mathrm{~m} / \mathrm{s}$ \\
\hline
\end{tabular}


There are analytical approaches given in the literature which may be suitable for the determination of the elements of the acoustic network in Fig. 7. E.g. Lerch et al. [7] provide an approach for the calculation of the mass elements $M_{\mathrm{ac}, \mathrm{V} 1 / 2}$. There the vibrating air mass on one side of the a piston in an infinity large wall is calculated by

$$
M_{\text {ac, V,piston }}=2 \cdot r_{\mathrm{pl}} \frac{\rho_{0}}{A_{\text {piston }}} \cdot \frac{8}{3 \pi}
$$

for low frequencies, where $A_{\text {piston }}$ is the area of the piston. Lenk et al. [3] describe the acoustic compliance of an air cavity by the equation

$$
N_{\mathrm{ac}, \mathrm{V}}=\frac{V}{\kappa \cdot p_{0}},
$$

where $\kappa$ is the adiabatic coefficient and $p_{0}$ is the acoustic normal pressure of the air. Further Lenk et al. deliver analytically relations for $Z_{\mathrm{ac}, \mathrm{h}}$ and $M_{\mathrm{ac}, \mathrm{h}}$ for the acoustic behavior of air in a channel. The vibrating air masses in front and rear of the several holes take part, too. The amount of this air masses are regarded in the orifice correction which describes the virtually extension of an air channel. An analytical description of the orifice correction is given by Skudrzyk [5]. Mechel [4] proposes an approach for the calculation of the vibrating air masses of perforated plates.

However, all of these analytically approaches consist of boundary conditions which do not match the setup of the encapsulated plate transducer to be analyzed. Hence the acoustical network elements have to be determined by FE-simulation experiments, too.

The determination of the acoustic elements in Fig. 7 needs to distinguish between a plate with and without perforation. The network of a plate with perforation is defined by the mass elements $M_{\mathrm{ac}, \mathrm{h}}$ and $M_{\mathrm{ac}, \mathrm{V}}$ in a frequency range

$$
f \ll f_{0, \mathrm{ac}}=\frac{1}{2 \pi \sqrt{M_{\mathrm{ac}, \mathrm{h}} \cdot N_{\mathrm{ac}, \mathrm{V}}}} .
$$

Using harmonic simulation experiments the mass $M_{\mathrm{ac}, \mathrm{h}}$ can be calculated by the ratio of the air pressure at the end of the air cavities $\underline{p}_{\mathrm{v}}$ to the volume flow. It yields

$$
M_{\mathrm{ac}, \mathrm{h}}=\frac{1}{j \omega} \cdot \frac{\underline{p}_{\mathrm{v}}}{\underline{q}} .
$$

Using equation (6) the acoustic compliance $N_{\text {ac, } \mathrm{V}}$ can be determined by a modal analysis. Based on equation (7) the mass of the air in the cavities $M_{\mathrm{ac}, \mathrm{V}}$ can be determined using the pressure at the plate itself by

$$
M_{\mathrm{ac}, \mathrm{V}}=\frac{1}{j \omega} \cdot \frac{\underline{p}_{\mathrm{pl}}}{\underline{q}}-M_{\mathrm{ac}, \mathrm{h}} .
$$

The acoustic pressure at the bending plate $\underline{p}_{\mathrm{pl}}$ is not constant across the whole surface. To get correct results an equivalent mean pressure $\underline{p}_{\text {pleq }}$ is used. The pressure is weighted according to the displacement of the plate. It is calculated by

$$
\underline{p}_{\mathrm{pl}, \mathrm{eq}}=\frac{\sum_{\mathrm{i}} \underline{p}_{\mathrm{pl}, \mathrm{i}} \cdot \underline{\xi}_{\mathrm{i}} \cdot A_{\mathrm{i}}}{\sum_{\mathrm{i}} \underline{\xi}_{\mathrm{i}} \cdot A_{\mathrm{i}}} .
$$

For a plate without perforation the network in Fig. 7 consists only of the elements $N_{\mathrm{ac}, \mathrm{V}}$ and $M_{\text {ac, } \mathrm{V}}$. In this case the acoustical compliance can be determined by the equation

$$
N_{\mathrm{ac}, \mathrm{V}}=\frac{1}{j \omega} \cdot \frac{\underline{q}}{\underline{p}} .
$$

A modal experiment and the equation

$$
M_{\mathrm{ac}, \mathrm{V}}=\frac{1}{\omega^{2} N_{\mathrm{ac}, \mathrm{V}}}
$$

give the according acoustic masses in the front and rear of the plate.

Simulations with different perforations showed a dominant influence of $M_{\mathrm{ac}, \mathrm{h}}$ on the behavior of the acoustic network in Fig. 7, especially the pressure $p_{\mathrm{V}}$. In most cases it is sufficient to simulate the acoustic interactions between the air cavities and the plate transducer using this mass element exclusively. It would be an advantage to have an analytically description for this element, based on the number of holes and the hole size. This would spare the previous described complex FE-analyses.

Fig. 15 shows the normalized mass $M_{\mathrm{ac}, \mathrm{h}} / M_{\mathrm{ac}, \mathrm{h} 100 \%}$ for different perforation fractions

$$
y_{\mathrm{a}}=N \cdot\left(\frac{r_{\mathrm{h}}}{r_{\mathrm{pl}}}\right)^{2}
$$

and hole radiuses $r_{\mathrm{h}} . M_{\mathrm{ac}, \mathrm{h} 100 \%}$ describes the fictive mass for a plate which consists only of holes. The value of $M_{\mathrm{ac}, \mathrm{h} 100 \%}$ is defined by the geometry of the plate. 


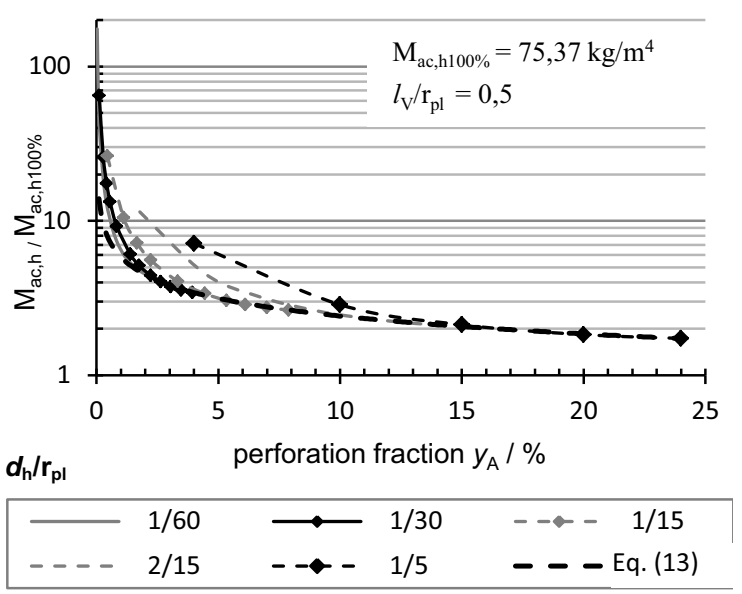

Fig. 9 Normalized acoustic Mass of the perforation for different perforation configurations

An approach for the calculation of $M_{\mathrm{ac}, \mathrm{h} 100 \%}$ is given by

$$
\begin{aligned}
& M_{\mathrm{ac}, \mathrm{h}, 100 \%}=\rho_{0} \cdot \frac{2 l_{100 \%}}{\pi r_{\mathrm{pl}}{ }^{2}}, \\
& \text { for } l_{100 \%}=0,43 \cdot r_{\mathrm{pl}}
\end{aligned}
$$

This approach is equal to the calculation of the acoustic mass in a channel (Lenk et al. [3]). The behavior of the acoustic mass $M_{\mathrm{ac}, \mathrm{h}}$ according to Fig. 15 can be described approximately by the developed equation

$$
M_{\mathrm{ac}, \mathrm{h}}=M_{\mathrm{ac}, \mathrm{h}, 100 \%} \cdot\left(N \cdot \frac{r_{\mathrm{h}}}{r_{\mathrm{pl}}}\right)^{-0,383} .
$$

Equation (13) shows that the perforation fraction has to be multiplied by factor 6 to halve the acoustic mass $M_{\mathrm{ac}, \mathrm{h}}$ and consequently the pressure $p_{\mathrm{V}}$ (eq. 7).

To ensure an acoustical transparency of the plate transducer in the capsule where the air cavity length is $l_{\text {cav }}=r_{\mathrm{pl}}$, the hole size has to be $r_{\mathrm{h}}=r_{\mathrm{pl}} / 20$ and the number of holes $N$ as high as possible.

The network approaches in Fig. 6 and Fig. 7 have been proven valid by Krause and Steinert [9], [10] up to the first acoustical and mechanical resonance frequency. The network models allow the dynamic system simulation within seconds in a network analyzer program, e.g. LTSpice. A full dynamic analysis of the plate transducer using FE-methods needs a computing time of a couple of hours using an advanced todays PC.

\section{Summary}

In this paper an approach for modeling a modified piezoelectric plate transducer has been presented. The developed model incorporates for the electrical, mechanical and acoustical behavior of the transducer. The elements of the network have been determined using FE-simulation experiments. The network approach allows a forecast of the dynamic behavior of the transducer up to the first resonance frequency, which is sufficient in the considered application. Future work concerns the suitability of the transducer for actuating or vibration reduction applications in microelectromechanical systems.

\section{References}

[1] Starke, E.; Marschner, U.; Pfeifer, G.; Fischer, W.-J. \& Flatau, A., "Combining Network Models and FE-Models for the Simulation of Electromechanical Systems", SPIE Conf. 7977 Smart Structures and Materials, 2011

[2] Starke, E.; Marschner, U.; Pfeifer, G. \& Fischer W.-J., ,Simulation-Based Optimization of a Piezoelectric Sound Generator by Combining a Finite-Element and a Network Model",

Proceedings of the ASME 2011 Conference on Smart Materials, Adaptive Structures and Intelligent Systems, SMASIS2011, Scottsdale, Arizona, USA, September 18-21, 2011

[3] Lenk, A., Ballas, R.G., Werthschützky, R., Pfeifer, G., "Electromechanical Systems in Microtechnology and Mechatronics", Springer, 2010

[4] F.P. Mechel, Schallabsorber, Bd.2, Innere Schallfelder, Strukturen, Stuttgart: Hirzel, 1995

[5] E. Skudrzyk, Die Grundlagen der Akustik, Springer, 1954

[6] M. Heckl und H.A. Müller, „Taschenbuch der Technischen Akustik“, Springer-Verlag GmbH, 1995

[7] R. Lerch, G. Sessler, und D. Wolf, „Technische Akustik: Grundlagen und Anwendungen", Berlin, Heidelberg: Springer Berlin Heidelberg, 2009

[8] Piezotechnology, Piezokeramische Materialien und Bauelemente - Grundlagen, Eigenschaften und Anwendungen, URL: http://www.piceramic.de (Date: 07/06/2012)

[9] Krause, M., Steinert, D., Starke, E., Marschner, U., Pfeifer, G. and Fischer, W.-J., "Piezoelectric Two Layer Plate for Position Stabilization", Proceedings of the ASME 2011 Conference on Smart Materials, Adaptive Structures and Intelligent Systems, SMASIS2012, Stone Mountain, Georgia, USA, September 19-21, 2012

[10] Steinert, D., „Elektrisch-mechanisch-akustische Wandlereigenschaften gelochter piezoelektrischer Biegeplatten für die Anwendungen in mikroelektromechanischen Systemen“, Akademikerverlag, 2012 